\title{
Unravelling the Fast Photocyclisation Reaction Mechanism(s) of 2-Benzoylpyridine in Aqueous Solvent by Time-resolved Spectroscopy
}

\author{
$\underline{\text { Yong Du }}^{\text {a,b }}$, Jiadan Xue ${ }^{\text {a,\#}}$, Mingde $\mathrm{Li}^{\text {a }}$, Xiangguo Guan ${ }^{\mathrm{a}}$, David W. \\ McCamant ${ }^{b}$ and David Lee Phillips ${ }^{a, *}$ \\ ${ }^{a}$ Department of Chemistry, The University of Hong Kong, Pokfulam Road, Hong Kong, P. R. China \\ ${ }^{b}$ Department of Chemistry, The University of Rochester, Rochester, New York, 14627 \\ \# Department of Chemistry, The Ohio State University, Columbus, Ohio, 43210
}

The photochemistry and photophysics of benzophenone (BP) as well as its derivatives have been the subjects of many studies due to their importance for understanding the photosensitizer mechanisms of electron transfer and hydrogen abstraction in the lowest triplet electronic excited states. ${ }^{[1-3]}$ Benzoylpyridines (BPy) are similar to BP and may be employed as potential photosensitizers due to their better water solubility than BP and they are particularly interesting since they contain heteroaromatic ring(s) with the nitrogen atom able to appear at different positions in the molecule. The different positions of $\boldsymbol{N}$-substituents within the aromatic rings can significantly change the photophysical properties and photochemical reactivity of the BPy $n \pi^{*}$ triplet state. ${ }^{[2,3]}$ By studying 2-BPy, 3-BPy and 4-BPy (where the number represents the $\mathbf{N}$ atom position within the aromatic rings) in different solvents with steady-state and laser flash photolysis measurements, Favaro and coworkers found that the lifetimes and reactivity of the triplet states 3-BPy and 4-BPy are very similar to those of $\mathrm{BP}^{[2]}$ while they observed appreciable differences and an unusual photodegradation behavior for 2-BPy. ${ }^{[3]}$ The fast intramolecular photocyclisation reaction occurs for 2-BPy in solvents that have H-bonding ability, but the reaction mechanism(s) for this reaction appears quite complex and controversial.

A combined femtosecond transient absorption (fs-TA) and nanosecond timeresolved Resonance Raman (ns- $\mathrm{TR}^{3}$ ) spectroscopic investigation of the photoreaction of 2-benzoylpyridine (2-BPy) in acetonitrile, neutral, basic and acidic aqueous solvents is reported. ${ }^{[4]}$ Fs-TA results showed that the $n \pi^{*}$ triplet 2-BPy is the precursor leading to the photocyclisation reaction in neutral and basic aqueous solvents. The cis triplet biradical and the cis singlet zwitterionic species produced during the photocyclisation reaction were initially characterized by $n s-\mathrm{TR}^{3}$ spectroscopy. In addition, a new species was uniquely observed in basic aqueous solvent after the cis singlet zwitterionic species decays and this new species was tentatively assigned to the photocyclised radical anion. The ground conformation of 2BPy in acidic aqueous solvent is the pyridine-nitrogen protonated 2-BPy cation (2- 
BPy-NH ${ }^{+}$) rather than the neutral form of 2-BPy. After laser photolysis, the singlet excited state $\left(\mathrm{S}_{1}\right)$ of $\mathbf{2}-\mathbf{B P y}-\mathbf{N H}{ }^{+}$is generated and evolves through excited state proton transfer (ESPT) and efficient intersystem crossing (ISC) processes into the triplet exited state $\left(\mathrm{T}_{1}\right)$ of the carbonyl-oxygen protonated 2-BPy cation $\left(\mathbf{2}-\mathbf{B P y}-\mathbf{O H}^{+}\right)$and then photocyclizes with the lone pair of the nitrogen involved in the heterocyclic ring. Cyclisation reactions take place both In neutral/basic and acidic aqueous solvents, but the detailed photocyclisation mechanism (shown in SCHEME1) in these two different aqueous solvents is very different. This is likely due to the different precursor conformation and the H-bonding influence from the solvent on the reactions.

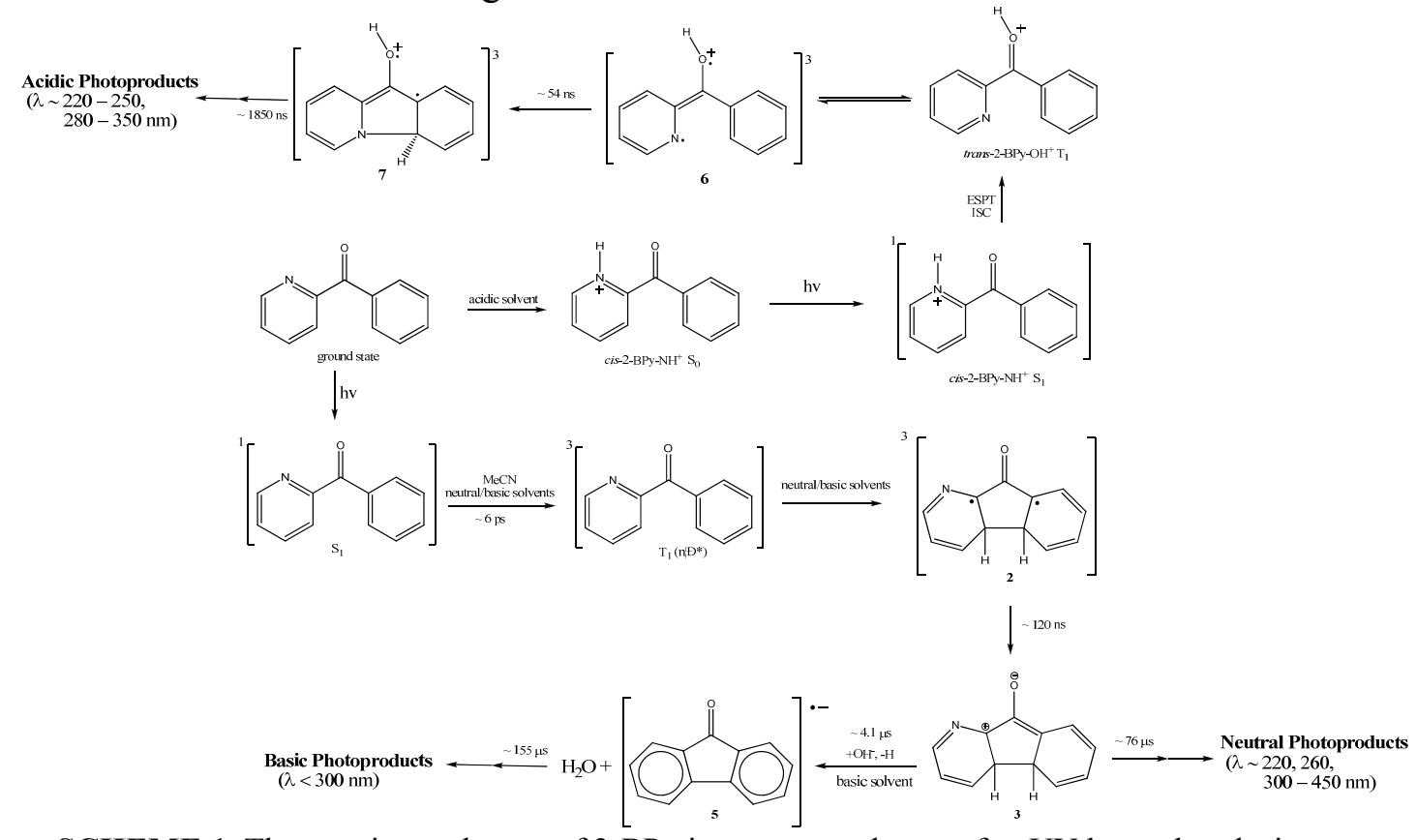

SCHEME 1. The reaction pathways of 2-BPy in aqueous solvents after UV laser photolysis

\section{ACKNOWLEDGMENTS}

This work was supported by a grant from the Research Grants Council of Hong Kong (HKU 7035/08P and HKU 1/01C), the award of a Croucher Foundation Senior Research Fellowship (2006-07) from the Croucher Foundation and an Outstanding Researcher Award (2006) from the University of Hong Kong to DLP. Work performed at the University of Rochester was supported by start-up funding to DWM.

\section{REFERENCES}

1. C. R. Hurt, N. Filipescu, J. Am. Chem. Soc. 94, 3649-3651(1972).

2. F. Ortica, F. Elisei, G. Favaro, J. Chem. Soc., Faraday Trans. 91, 3405-3413(1995).

3. P. Bortolus, F. Elisei, G. Favaro, S. Minti, F. Ortica, J. Chem. Soc., Faraday Trans. 92, 18411851(1996).

4. Y. Du, J. Xue, X. G. Guan, M. Li, D. W. McCamant, D. L. Phillips Chemistry-A European Journal., (2010) In Revision. 